\title{
Features of Operation of Bifilar Cooper and Tesla Coils in Pulse Regime
}

\author{
V. L. Busov, Yu. V. Vasiliev \\ Donbass State Engineering Academy, Kramatorsk, Ukraine \\ Email: vlbusov49@gmail.com
}

How to cite this paper: Busov, V.L. and Vasiliev, Y.V. (2021) Features of Operation of Bifilar Cooper and Tesla Coils in Pulse Regime. Applied Mathematics, 12, 1156-1165. https://doi.org/10.4236/am.2021.1212074

Received: October 23, 2021

Accepted: December 7, 2021

Published: December 10, 2021

Copyright $\odot 2021$ by author(s) and Scientific Research Publishing Inc. This work is licensed under the Creative Commons Attribution International License (CC BY 4.0).

http://creativecommons.org/licenses/by/4.0/

\begin{abstract}
The characteristic features of operation in a pulsed regime of bifilar Cooper and Tesla coils during magnetic pulse processing and heating of the environment are considered using the dependence of the maximum amplitude at the leading edge of the magnetic pulse $H_{\max }^{\text {out }}$ on its time duration $\tau$. The spatial distribution of the magnetic induction lines $\boldsymbol{B}$ inside and around the Cooper coil is given, where, unlike the Tesla coil, the maximum values of $\boldsymbol{B}$ arise in the extreme planes of the coil, and its central plane is zero. The defining advantages of new methods of magnetic pulse processing and heating of the environment are considered: the dependence $H_{\max }^{\text {out }}(\tau)$ at $\tau \rightarrow 0$; the exchange of energy between the magnetic pulse and the environment; periodic sequence of series of unipolar magnetic pulses at various processing modes. It is shown that 1 ) for electric fields $E^{\text {out }}=10^{10}-10^{12} \mathrm{~V} / \mathrm{m}$, arising at the leading edge of a magnetic pulse with duration $10^{-5}-10^{-7} \mathrm{~s}$, in the deformation fractal space the electrodynamics of these fields is nonlinear; 2 ) inside the Tesla coil, in the package of conducting tapes and tubes, the pumping energy from the pulsed field $E^{\text {out }}$ is mainly dissipated in the skin layer in the form of refractory coatings and heats the environment at all stages of plastic deformation of workpieces and heating elements up to the collapse of unstable microcracks. The coefficient of conversion of the pump energy in the pulsed regime with respect to the constant current regime is found.
\end{abstract}

\section{Keywords}

Cooper and Tesla Bifilar Coils, Leading Edge of a Magnetic Pulse, Deformation Fractal Space, Nonlinear Electrodynamics

\section{Introduction}

It is known that during static and cyclic deformation of samples, details and 
various structures, the latent component of the energy supplied to the sample does not exceed several percent of the total energy expended ([1], p. 250), the rest of it turns into heat, dissipates in the environment and heats the sample [2]. When two bodies collide, the kinetic energy of the impinging body is also mainly converted into thermal energy and the radiation energy of the atoms of the colliding bodies. It should be noted here that such processes take place not only when different bodies are in direct contact, but also when bodies are exposed to an electromagnetic field [3] [4] [5] [6] [7], the photons of which are accelerated by light electrons scattered by heavy cations of bodies with the release of Joule heat.

It is known ([8], p. 275) that when direct currents pass through conductors, the release of Joule heat obeys the Joule-Lenz law

$$
Q=\frac{J^{2}}{\sigma}=\frac{\sigma}{e^{2}} \boldsymbol{F}^{2},
$$

where $Q$ is the amount of Joule heat released in a unit of volume per unit of time $\left[\mathrm{J} /\left(\mathrm{m}^{3} \cdot \mathrm{s}\right)\right]$; $J$ is current density $\left[\mathrm{A} / \mathrm{m}^{2}\right] ; \sigma$ is electrical conductivity of the conductor material $[\Omega \cdot \mathrm{m}]^{-1} ; e$ is the electron charge; $\boldsymbol{F}$ is force acting on the current carrier with charge $e, \boldsymbol{F}=e \cdot \boldsymbol{E}^{\text {out }}$. For technical alternating currents varying rather slowly in time $t$, the expression $Q$ retains the form (1). If the dependence $J(t)$ is given, then integration over time makes it possible to find the exact value of $Q$, while the mechanism of the transition of the kinetic energy of the ordered motion of conduction electrons to the vibrational energy of atoms at the sites of the crystal lattice during the scattering of these electrons by the nuclei of the site ions remains unchanged.

It is known ([9] [10], p. 462) that in strong electromagnetic fields most of the atoms or molecules in dielectrics and semiconductors are in an excited state and can no longer absorb electromagnetic waves. Here nonlinear effects such as frequency doubling and tripling occur, i.e. the appearance of double and triple harmonics, confirmed experimentally. To describe a nonlinear medium in strong fields, the strengths of the external field $E^{\text {out }}$ are compared with the strength of the intra-atomic field $E_{a t} \cong e / a^{2} \cong 10^{10} \div 10^{11} \mathrm{~V} / \mathrm{m}$. At $E^{\text {out }} \ll E_{a t}$, each atom is taken as a harmonic oscillator. When $E^{\text {out }} / E_{a t} \leq 1$, the harmonism of the atomic oscillator is violated, and the oscillator becomes anharmonic. Here, the dependence of the interaction energy of a strong field with a medium ceases to be quadratic, i.e. the dielectric susceptibility $\chi$ itself depends on $E^{\text {out }}$. In this case, the polarization $\boldsymbol{P}$ of a unit volume of the medium can be expanded in a series in terms of the parameter $E=E^{\text {out }} / E_{a t}$.

$$
P=\chi^{(1)} \cdot E+\chi^{(2)} \cdot E^{2}+\chi^{(3)} \cdot E^{3}+\cdots,
$$

where the coefficients $\chi^{(1)}, \chi^{(2)}, \chi^{(3)}$ etc., it is customary to call nonlinear susceptibilities, which are equal in order of magnitude $\chi^{(1)} \sim 1 / E_{a t} ; \chi^{(2)} \sim 1 / E_{a t}^{2}$. According to [9], the magnetization $\mathrm{M}$ can also be represented using the corresponding magnetic characteristics. 
At present, Tesla [11] and Cooper [12] bifilar coils are widely used in magnetic systems. Both coils of parallel winding contain two parallel wires in most cases of the same cross section, and in the Cooper coil the ends of the wires En1 and En2 are either soldered together or in a contact patch in the form of a disk, and their beginnings Bgn1 and Bgn2 are free and connected to a power source, in our case to the pulse generator. On the contrary, in a Tesla coil, the wiring diagram is as follows: Bgn1 $\rightarrow$ En2; Bgn2 $\rightarrow$ En1. In other words, in a Cooper coil, free leads are always on one side, and in a Tesla coil-from different sides; in a Tesla coil, currents in adjacent turns are directed in one direction, in a Cooper coil, currents in adjacent turns flow in opposite directions.

For more than half a century, magnetic pulse processing has been used mainly for axisymmetric details made of non-ferrous $\mathrm{Cu}$-based alloys, while the mechanism of nucleation of dislocation structures under the influence of MP and the subsequent interaction of MP with such structures remained unrevealed. The use of discrete and plasma models of plasticity and fracture [3] [4] [5] [6] [7] makes it possible to reveal this mechanism and can radically change the approaches when choosing magnetic concentrators such as coils and magnetic pulse generators with a fundamentally new set of control parameters.

The purpose of this work is to theoretically substantiate the processes of magnetic pulse processing and heating of the environment using Cooper and Tesla coils loaded in a pulsed regime, and a possible design solution for these processes.

\section{Theoretical Model}

Under shock loads, shock waves appear in materials (metals, semiconductors, dielectrics), which, in the presence of impurity ions, lead to the appearance of an intermittent field that causes the generation of linear defects-dislocations and subsequent plastic deformation [3] [4] [5] [6]. An external magnetic field pulse $\boldsymbol{H}^{\text {out }}$ can also be considered as a shock load. The volumetric energy density of an individual magnetic pulse (MP) $u_{\text {mim }}$, propagating along the normal to the free surface, can be represented as an integral over time $t$ and transformed using the mean theorem

$$
u_{\text {mim }}=\frac{\mu_{0} \cdot \mu}{2 \cdot T_{\text {rep }}} \int_{0}^{\tau}\left[H^{\text {out }}(t)\right]^{2} \mathrm{~d} t=\frac{k_{u} \cdot \mu_{0} \cdot \mu}{2}\left(H_{\max }^{\text {out }}\right)^{2} \cdot \frac{\tau}{T_{\text {rep }}},
$$

where $\mu_{0}$ is the magnetic constant; $\mu$ is magnetic permeability of the material; $2 \pi \cdot\left(T_{\text {rep }}\right)^{-1}$ is MP repetition rate; $\tau$ is the time duration of MP; $H_{\max }^{\text {out }}$ is maximum amplitude $H^{\text {out }}$ at the leading edge of the MP; $k_{u}$ is numerical coefficient, $0<k_{u}<1$. For $u_{\text {mim }}=$ const and $T_{\text {rep }}=$ const the dependence of $H_{\max }^{\text {out }}$ on $\tau$ in (3) is nonlinear:

$$
H_{\max }^{\text {out }}=\left(\frac{2 \cdot u_{\text {mim }} \cdot T_{\text {rep }}}{k_{u} \cdot \mu_{0} \cdot \mu}\right)^{1 / 2} \cdot \frac{1}{\tau^{1 / 2}},
$$

whose graph is confirmed empirically in a wide range of $\tau$ from $10^{-1} \mathrm{~s}$ to $10^{-7} \mathrm{~s}$ by three methods (machine generators, capacitor bank discharge, explosive me- 
thod) and is given in ([10], p. 662). Hence it becomes clear that at $\tau \rightarrow 0$ the values of $H_{\max }^{\text {out }}$ can reach superstrong fields $H_{s s}=10^{6} \div 10^{7}$ oerst $=7.96 \times\left(10^{7} \div 10^{8}\right) \mathrm{A} / \mathrm{m}$. It should be noted here that according to ([10], p.662), with short-term current pulses, the skin effect becomes significant: currents flow through the skin layer on the inner surface of the turns of the coils, which leads to an increase in the current density, while for a short time of the current pulse, the heat removal from the skin layer is negligible, and the process of heating the material of the turns of the coils, any conductors inside the coils, occurs adiabatically. The surface temperature of these conductors is $\approx 3 \times 10^{3} \times\left(H_{\text {max }}^{\text {out }}\right)^{2}$, which can lead to melting of the surface layer of conductors, even from refractory materials. Let us give an equation for the tensors of the electromagnetic field in vacuum $F_{k m}^{\text {out }}$ and in a deformed material $F_{k m}^{i f}$ [3].

$$
\frac{\partial\left(F_{k m}^{\text {if }}+F_{k m}^{\text {out }}\right)}{\partial x_{m}}=-\left(J_{k}^{\text {out }}+J_{k}^{\text {own }}\right),
$$

where $J_{k}^{\text {out }}$ and $J_{k}^{\text {own }}$ are 4-density vectors, respectively, of external and intrinsic electric currents in the deformed material. If $J_{k}^{\text {out }}$ is set in the form of rectangular pulses, then all components $F_{k m}^{\text {out }}$ in vacuum will take the same pulse shape, and in a deformed material this pulse shape always takes place for $J_{k}^{\text {own }}$ components and, accordingly, for $F_{k m}^{i f}\left(\boldsymbol{E}^{o w n}, \boldsymbol{H}^{\text {own }}\right)$ [4] [5] as for generalized space. As for the connection between the magnetic induction $\boldsymbol{B}$ and the electric field $\boldsymbol{E}$, it can be easily traced from Faraday's law

$$
\operatorname{rot} \boldsymbol{E}=-\frac{\partial \boldsymbol{B}}{\partial t},
$$

where with the help of [13] and the relation $E_{a}=c \cdot B_{a}$ in dielectrics and semiconductors it is possible to estimate the amplitude of the alternating electric field $E_{a}$ in MP at the MP amplitude $B_{a}$. At $\tau \rightarrow 0$ for superstrong fields $H_{\text {ss }}$, this estimate reaches the interval $E_{a}=10^{10} \div 10^{12} \mathrm{~V} / \mathrm{m}$ and higher. At the leading edge of the MP with duration $\Delta \tau_{f f}$, an intrinsic electric field $\boldsymbol{E}^{\text {own }} \perp \boldsymbol{H}^{\text {out }}$ arises, which in the first approximation can be represented by a rectangular pulse. Similarly, on the trailing edge of the MP, the same field of opposite sign takes place. In addition, expression (6) allows one to determine the occurrence of induction currents in a pulsed regime near the surface of the conductor in two cases. If $\boldsymbol{H}^{\text {out }} \perp$ of this surface, then the circulation of the induction current within the skin layer will oppose the external $\boldsymbol{H}^{\text {out }}$, creating its own magnetic field $\boldsymbol{H}^{\text {own }}$ towards the external one. Here, it is necessary to assume that MP has a rectangular shape with duration $\tau$, while the duration of the MP leading edge $\Delta \tau_{f f}$ and the relaxation time of conduction electrons $\tau_{\text {rel }}$ are small compared to $\tau: \Delta \tau_{f f} \ll \tau, \tau_{\text {rel }} \ll \tau$. For $t>\Delta \tau_{f f}$, the field $\boldsymbol{H}^{\text {out }}$ becomes unchanged in time, and $\boldsymbol{E}^{\text {own }}=0$, and therefore the concept of cyclotron frequency is applicable ([10], p. 846), when the Lorentz force and centrifugal force are equal, and the conduction electrons move along orbits of diameter $D$. At the trailing edge of the MP, the situation is repeated, but at $\tau=10^{-4} \div 10^{-7} \mathrm{~s}$ induc- 
tion currents arise in conductors within the skin layer with a thickness of $\delta=0.01-0.1 \mu \mathrm{m}$, and for $t>\Delta \tau_{f f} \quad D \approx 1 \div 10 \mu \mathrm{m}$. If $\boldsymbol{H}^{\text {out }} \|$ of the surface of the conductor, then the field $\boldsymbol{E}^{\text {own }}$ also takes place at the leading and trailing edges of the MP, but $\boldsymbol{E}^{\text {own }} \perp$ of the free surface. Here, the plane of circulation of induction currents is normal to the free surface, while the trajectories of electrons pass mainly outside the skin layer, but enter it for a short time. The interaction of MP with conduction electrons occurs in a strongly inhomogeneous electromagnet field, where $\boldsymbol{H}^{\text {out }}$ and $\boldsymbol{E}^{\text {own }}$ decrease exponentially $\exp (-z / \delta)$, and electric fields proportional to induction currents remain unchanged within the entire trajectory of electrons , and $D$ is limited by the thickness of the metal strip inside the coils.

Consider the spatial distributions of the lines of magnetic induction $\boldsymbol{B}$ around and inside the Tesla and Cooper coils. This distribution of the Tesla coil is identical in shape to the distribution of a conventional wire coil with current ([8], Fig. 29.5). For a Cooper coil using an ICs sensor of the SS490 series $\left(B_{\max }= \pm 800 \mathrm{Gs}\right)$ with a linear Hall effect and a calibration coefficient of $0.178 \mathrm{Gs} / \mathrm{mV} \equiv 1.78 \times 10^{-2} \mathrm{~T} / \mathrm{V}$, this distribution was found and shown in Figure 1 .

In this regard, it is of interest to consider the real structure of MP. Figure 2 shows the MP at $\tau=1.25 \mathrm{~ms}$ obtained by the authors using a magnetic pulse generator consisting of a rectifier unit with a filter capacitor $C_{1}$, a capacitor

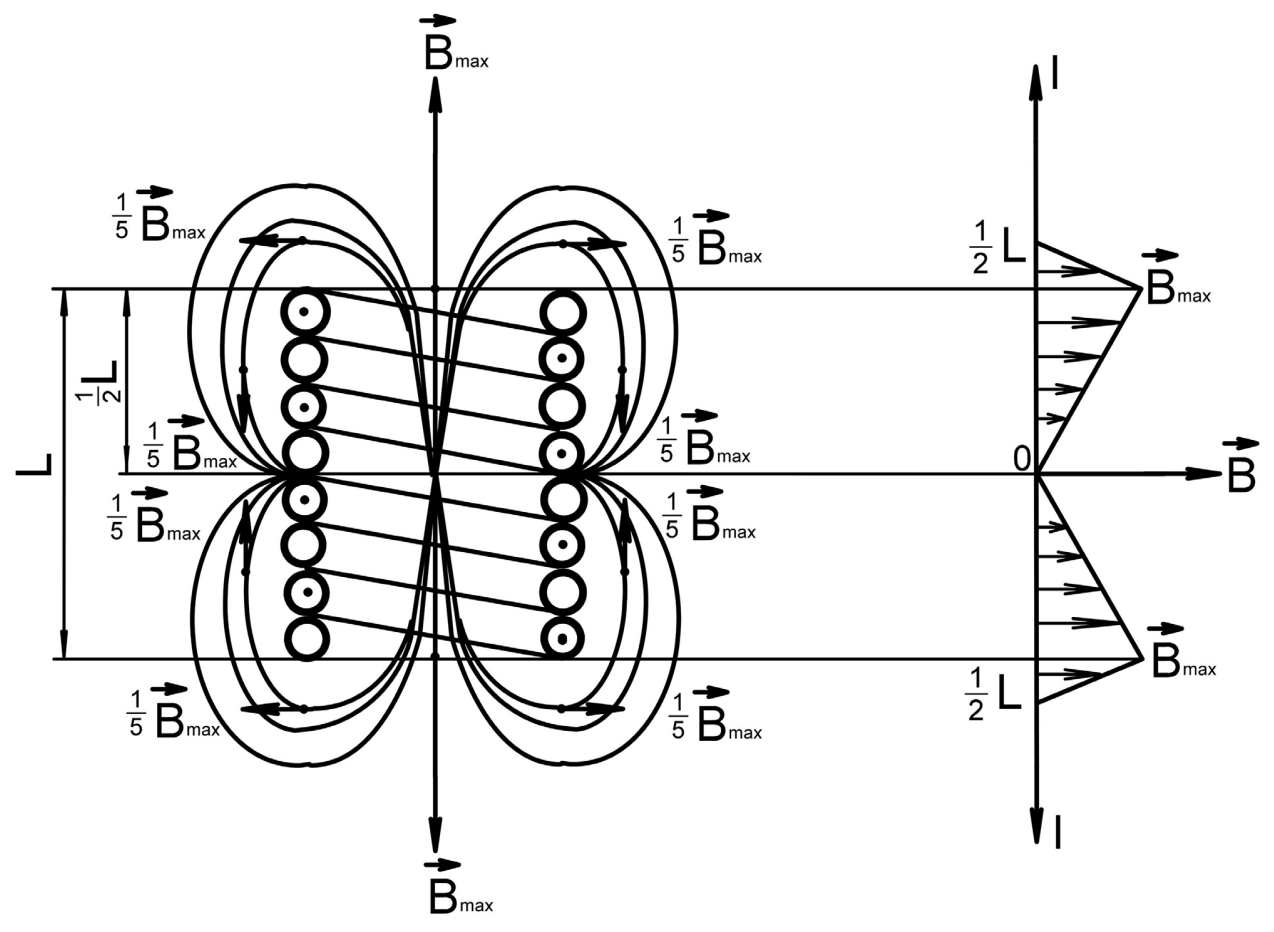

(a)

(b)

Figure 1. Spatial distribution of the lines of magnetic induction $\boldsymbol{B}$ for the Cooper coil. (a) the shape of the magnetic field of the Cooper coil; (b) distribution of $\boldsymbol{B}$ along the axis of the Cooper coil of length $L ; O O$-bifilar loops. 


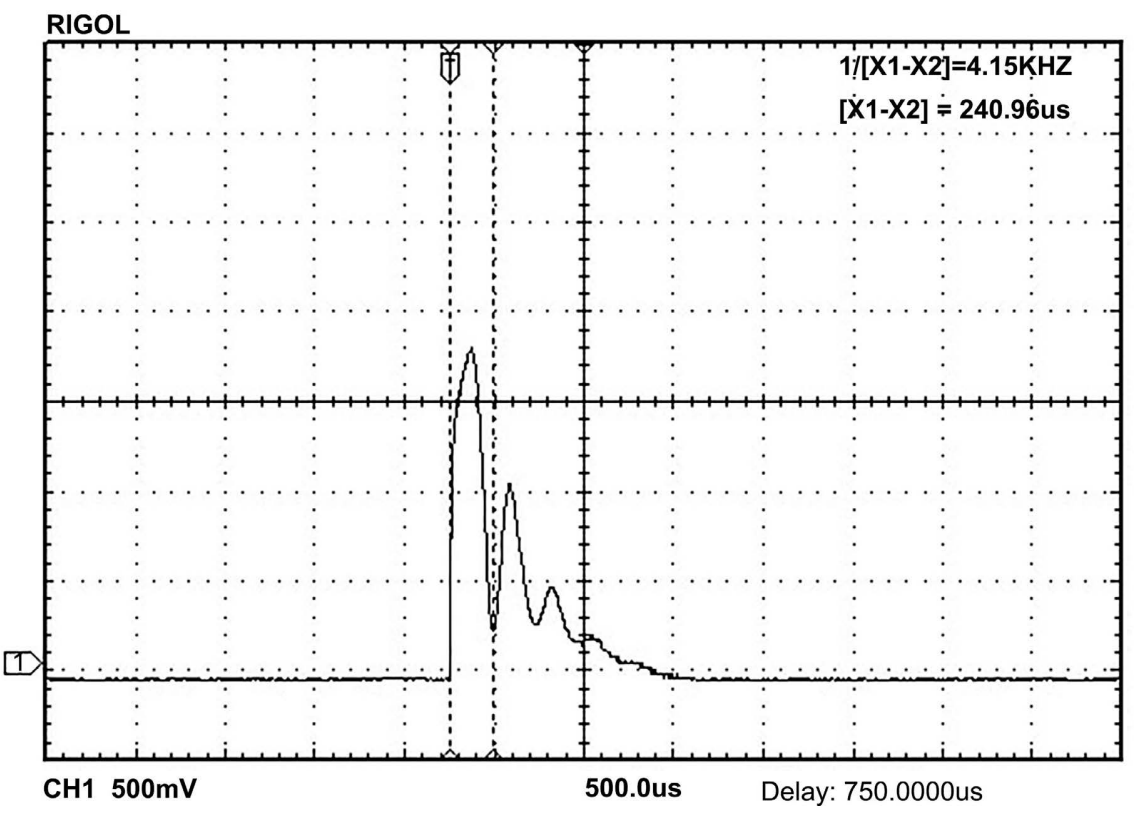

Figure 2. Unipolar magnetic pulse $V_{H}(t)$ with a duration of $1.25 \mathrm{~ms}$ with a maximum amplitude of the Hall voltage $V_{H}=1.8 \mathrm{~V}$. Scale division: $100 \mu$ s along the abscissa; on the ordinate axis $100 \mathrm{mV}$.

bank, a switching device of two thyristors $T_{1}$ and $T_{2}$ and a discharge capacitor $C_{2}$, a Tesla coil and an amplitude control unit and the duty cycle of the MP sequence. A unipolar pulsed field signal applied to the Tesla coil, obtained using a CYSJ362A Hall sensor (Technologies Gmb \& Co.KG, Germany), consists of three sections in the form of three peaks with descending branches, where in the first section the maximum coil current amplitude $I_{\max 1}$ of the first peak $4800 \mathrm{~A}$, and the duration of its leading edge $\Delta \tau_{f f 1}$ is $\Delta \tau_{f f 1}=70-75 \mu$ s, and in the second section $I_{\max 2}=2930 \mathrm{~A}, \Delta \tau_{\text {ff } 2} \approx 90 \mu \mathrm{s}$; in the third section $I_{\max 2}=1307 \mathrm{~A}, \Delta \tau_{f f 2} \approx 85 \mu \mathrm{s}$; on average $\Delta \tau_{f f}=0.08 \div 0.1 \mathrm{~ms}$. The background part of the pulse does not exceed $16 \%-19 \%$ of $I_{\max 1}, I_{\max 2}$. The differences between $I_{\max 1}$ and $I_{\max 2}, I_{\max 2}$ and $I_{\max 3}$ correspond to the losses for increasing the latent energy in the turns of the coils and conductors inside them and their heating. Here, MP was recorded using a DS5022M digital oscilloscope (RIGOL, China) and a Hall sensor with a conversion factor of the Hall voltage $V_{y}[\mathrm{mV}]$ into the coil current $I_{c}[\mathrm{~A}]$ equal to $200 \mathrm{~A} / 75 \mathrm{mV}$.

The dependence $H_{\max }^{\text {out }}(\tau)$ allows a completely new look at the entire process of processing materials by pressure, since it allows to distinguish two regimes: the regime of generation of defects at $\tau=10^{-7} \div 10^{-4} \mathrm{~s}$ ascending branch $H_{\max }^{\text {out }}(\tau)$ let us call it the regime "hammer", and the regime of formation products at $\tau=10^{-3} \div 10^{-1} \mathrm{~s}$ descending branch $H_{\text {max }}^{\text {out }}(\tau)$ or "press" regime. Hence, the first defining advantage of this method is the control of the plastic deformation process by varying $\tau$.

Another important advantage in the magnetic pulse processing of dielectrics, ferroelectrics and semiconductors is that a short MP exchanges energy between 
the region covered by MP moving through the material and the environment, while through the MP leading edge, which falls steeply in the direction of MP propagation, gives energy to the medium, and the trailing edge of the MIP, which falls more weakly in the opposite direction, takes it back; the momentum of the field is a reversible relationship "field-medium". It should be noted here that in the "press" regime it is necessary to use crossed fields: a constant magnetic field normal to the surface of the sheet material during sheet stamping and rolling, the direction of the magnetic field of the MP can be in the plane of the product or make an angle $45^{\circ}$ with this plane. In each specific case, an engineering technical solution is to find the angles of crossing of these fields and the angles between the directions of these fields and the normal to the surface of the product.

The third defining advantage of the method is the identification of a periodic sequence of MP series of the "hammer" and "press" type. Here, the engineering solution is to find the number of MP in series of the "hammer" $\left(N_{m}\right)$ and "press" $\left(N_{p r}\right)$ type at this stage of material hardening, i.e. finding the correct combination of the level of generation of defects and timely softening by redistributing the arising movable defects and thereby forming the size and shape of the product required by the technology. Such a nature of the impact on the deformation volume of the product is due to the level of Maxwell stresses $\sigma_{\alpha \beta}^{i f}$ in the defect cores, which depend on the strengths of the internal electric and magnetic fields, and the level of elastic stresses $\sigma_{i k}^{e l}$ in the medium surrounding the defect cores [5]. Indeed, in metals with $\sigma_{\alpha \beta}^{i f}>\sigma_{y s}$-the yield stress of the material by $10 \%$ $30 \%$, there is an increase in the density of mobile and sessile dislocations, which is equivalent to the strengthening of metals and their alloys, and when $\sigma_{\alpha \beta}^{i f} \cong \sigma_{y s}$, plastic deformation at the yield point. In dielectrics and semiconductors, such a site is absent, because immediately beyond the proportionality limit, brittle destruction occurs, since the dislocation density increases explosively.

In the case of $\sigma_{\alpha \beta}^{i f}>\sigma_{y s}$ between the "hammer" and "press" series, it is necessary to use electronic keys, which will inevitably lead to taking into account the inductance of the solenoid $L$, through which the current $I(t)$ flows. The main control parameters of the MP sequence during magnetic pulse processing are $\tau, \Delta \tau_{f f}, T_{\text {rep }}, N_{m}(t), N_{p r}(t), I(t)$.

Consider the use of bifilar coils in a pulsed mode when heating the environment, in particular water. The electric field arising at the leading edge of the MP can be represented by a rectangular pulse of duration $\Delta \tau_{f f}$. At the first stage of the theory, we neglect the influence of the trailing edge of the MP, which causes a negative jump of such a field in the opposite direction with subsequent exponential decay to zero. Faraday's law allows us to assume a constructive solution for a package of rods either from copper strips $\leq 1 \mathrm{~mm}$ thick, having a heat-resistant and refractory coating of beryllium or chromium bronze on both sides and twisted in the form of spirals, or a lattice of cylindrical copper tubes with the same coat- 
ings, fastened to a box-shaped profile or a profile in the form of a pipe.

Of particular interest is the determination of the conversion coefficient $k_{t r}$, which is equal to the ratio of the specific energy expended by the system in a pulsed regime to the analogous energy of the alternating technical current. Note that in a fractal deformed space, the magnetic and dielectric permittivities $\mu$ and $\varepsilon$ themselves depend on $H^{\text {out }}$ and $E^{\text {out }}$, respectively. Here these dependencies take the following form:

$$
\mu=k_{\mu} \cdot\left(H^{\text {out }}\right)^{\beta_{H B}-2} ; \varepsilon=k_{\varepsilon} \cdot\left(E^{\text {out }}\right)^{\beta_{H B}-2},
$$

where $\beta_{H B}$ is the Hausdorff-Besicovitch fractal dimension, $1<\beta_{H B} \leq 3 ; k_{\mu}$ and $k_{\varepsilon}$ are proportionality coefficients. According to [7] $\beta_{H B}$ is a function of the density of conduction electrons $n_{e}$. Hence, the dependence of the specific field energy in the material on $E^{\text {out }}, H^{\text {out }}$ can change from quadratic to cubic. A natural question arises: what volume is occupied by charged carriers in conductors at direct current, alternating current of frequency $f$ and at pulsed regime? With a constant current, this volume coincides with the volume of a conductor with a cross section $\mathrm{S}$ and a length $\mathrm{l}$. With alternating current, current carriers are located inside the surface skin layer with a thickness of $\delta=c \cdot(\sigma \mu f)^{1 / 2} ; c$ is the speed of light; $\sigma$ is electrical conductivity. In the pulsed mode $\tau=10^{-7} \div 10^{-3} \mathrm{~s}$ (ascending branch $H_{\max }^{\text {out }}(\tau)$ up to the inflection point), the volume of the skin layer falls mainly on refractory coatings of copper tubes and tapes thickness up to $100 \mu \mathrm{m}$. Here the volume occupied by current carriers is determined by the ratio $\frac{n_{p} \Delta \tau_{\text {ff }}}{\tau} \cdot((0.01 \div 0.1) \cdot S \cdot l)$, where $n_{p}$ is the number of peaks in the MP. Hence from (1) and (7) $k_{t r}$ for the magnetic field in conductor tape $1 \mathrm{~mm}$ thick is equal to

$$
k_{t r}=\frac{(0.01 \div 0.1) \cdot k_{\mu}}{\sigma} \cdot \frac{n_{p} \Delta \tau_{f f}}{\tau} \cdot\left(H^{\text {out }}\right)^{\beta_{H B}\left(n_{e}\right)-2} .
$$

Similarly, for the electric field $E^{\text {out }}$ in (8), the coefficient $k_{\mu}$ must be replaced by $k_{\varepsilon}$.

\section{Discussion of Results}

At the end of the 19th century, N. Tesla showed that the magnetic field inside his bifilar coil is $10 \%-20 \%$ higher than in a single-wire winding coil. After 77 years, W. Cooper proposed a coil in the form of a wave line wound on a cylinder of length $l_{s}$ or in a flat design. As follows from Figure 1, inside the cylindrical Cooper coil there is a magnetic field gradient along the solenoid axis, while on the extreme planes of the solenoid the magnetic field of the coil is maximal, but directed from the coil in opposite directions, and in its central plane the field is zero. On the contrary, inside the Tesla coil, this field is maximal and has no gradient along the axis, and decreases significantly on the extreme planes of the solenoid. In other words, the distribution of the lines of magnetic induction of the Cooper coil takes the shape of a "butterfly", the lines of "wings" of which form 
four independent circuits of the circulation of the magnetic field $\boldsymbol{H}_{s c}$, the current density in these circuits is zero, and the currents in the bifilar wires are not equal to zero, but directed in opposite directions. Wherein in this case there are flows of the electric field $\boldsymbol{E}_{S C}$ through all the circuits in different directions according to Maxwell's equation or the law of total current (Ampere's law) ([14], p. 25)

$$
\operatorname{rot} \boldsymbol{H}_{s c}=\boldsymbol{j}_{s c}+\frac{\partial \varepsilon \cdot \varepsilon_{0} \boldsymbol{E}_{S C}}{\partial t},
$$

where $\boldsymbol{j}_{s c}=0 ; \varepsilon_{0}$ is dielectric constant; $\varepsilon$ is dielectric constant. Such a distribution of $\boldsymbol{B}$ of the Cooper coil in a flat design can make it possible to efficiently perform magnetic-pulse processing of details such as sheets, plates, etc.

Of interest is the MP structure, which consists of three peaks, the leading edges of which have approximately the same duration $\Delta \tau_{\text {ff }} \approx 75 \div 90 \mu \mathrm{s}$. Here the first peak allows you to work in the hammer regime, and the third peak is closer to the press regime. Therefore, it is important to learn how to adjust the shape of the envelope of the peaks and their number. It should be noted that when choosing water as the environment: a weak electrolyte with a certain separation into hydrogen ions or protons $\mathrm{H}^{+}$and hydroxide ions $\mathrm{OH}^{-}$, this separation is usually estimated by the $\mathrm{pH}$ value-negative decimal logarithm of the proton concentration $\left[\mathrm{H}^{+}\right]$, allowing you to select acidic $(\mathrm{pH}<7)$ neutral and $(\mathrm{pH}=7)$ alkaline $(\mathrm{pH}>7)$ environment. The imposition of an MP and, accordingly, the field $E^{\text {out }}$ on it accelerates protons, which collide with metal targets such as membranes, partitions, contact spots in the form of disks, packs of ribbons and tubes and heat them up.

The analysis shows that: 1 ) the interval of MP durations $\tau=10^{-4} \div 10^{-1} \mathrm{~s}$ can be carried out by means of various types of thyristor generators, which corresponds to a wide range of values of the yield strengths of metals and alloys; at $\tau=10^{-9} \div 10^{-5} \mathrm{~s}$, usually Marx generators are used [15] [16] - high voltage pulse generators consisting of a cascade of capacitors connected in parallel through resistors, the discharge of which is performed with using gas dischargers-trigatrons , allowing for electrohydraulic processing and crushing of materials; 2) the most acceptable deforming elements inside bifilar coils when the environment is heated in a pulsed mode can be copper tubes with a diameter of $1-2 \mathrm{~mm}$, spirals of copper tapes up to $1 \mathrm{~mm}$ thick with refractory and heat-resistant coatings. Switching to a cheaper aluminum base and matching heat-resistant and refractory coatings will make domestic and commercial water heaters economically viable.

In our opinion, the use of Tesla and Cooper bifilar coils loaded with magnetic pulse generators makes it possible at the first stage to combine traditional drawing and rolling methods with magnetic pulse processing, and at the second stage to gradually abandon expensive and metal-intensive hydraulic presses and various types of hammers as sources of extremely harmful effects on the foundations of nearby buildings and an unacceptable noise level of the order of several tens 
of $\mathrm{db}$.

\section{Conflicts of Interest}

The authors declare no conflicts of interest regarding the publication of this paper.

\section{References}

[1] Honeycombe, R.W.K. (1968) The Plastic Deformation of Metals. Edward Arnold, Cambridge.

[2] Panin, V.E., Fedorov, V.V., Romashov, R.V., Khachaturyan, S.V. and Korshunov, V.Y. (1989) The Phenomenon of Structural and Energy Analogy of the Processes of Mechanical Destruction and Melting of Metals and Alloys. On Sat. Synergetics and Fatigue Fracture of Metals. Metallurgy, Moscow.

[3] Busov, V.L. (2019) Dynamic Equations of Evolution in Nuclei of Linear Defects of Crystalline Materials upon Collision of Solids. Physical Mesomechanics, 22, 91-96.

[4] Busov, V.L. (2020) On Separation of Charges and Formation of Linear Structures in the Nuclea of Dislocations in Metals. Applied Mathematics, 11, 739-752. https://doi.org/10.4236/am.2020.118049

[5] Busov, V.L. (2020) On the Relationship of the Discrete Model of the Nuclei of Line and Planar Defects and Continuum Models. Applied Mathematics, 11, 862-875. https://doi.org/10.4236/am.2020.119056

[6] Busov, V.L. and Grechkina M.V. (2020) Plasma Model of Generation and Slip of Linear Defects in Crystalline Materials. Applied Mathematics, 11, 1167-1177. https://doi.org/10.4236/am.2020.1111079

[7] Busov, V.L. (2021) Discrete Model of Plasticity and Failure of Crystalline Materiels. Applied Mathematics, 12, 147-156. https://doi.org/10.4236/am.2021.123010

[8] Kuzmichev, V.E. (1989) Laws and Formulas of Physics. Directory. Naukova Dumka, Kyiv.

[9] Bloembergen, N. (1965) Nonlinear Optics (A Lecture Note). W.A. Benjumin, Inc., New York.

[10] Physical Encyclopedic Dictionary (1984) Ch. ed. Prokhorov, A.M. Soviet Encyclopedia, Moscow.

[11] US 512,340. Dragon, s Lord. Patented January 9, 1894, N. Tesla.

[12] US 3,610,971. Bifilar Non-Inductive Winding. Patented October 5,1971, W. Cooper.

[13] Abramov, V.S., Busov, V.L. and Bityutskaya, L.A. (2010) On the Mechanism of Displacement of Point Defects in Alkali Halide Crystals under the Influence of a Pulsed Magnetic Field. Condensed Media and Interphase Boundaries, 12, 319-325.

[14] Tables of Physical Quantities (1976). Ch. ed. Kikoin, I.K. Atomizdat, Moscow.

[15] Fryungel, F. (1965) Impulse Technique. Generation and Application of Capacitor Discharges. Transl. with Germ. Moscow, L.

[16] Bulan, V., et al. (1999) High-Voltage Nanosecond Marx Generator with Quasi-Rectangular Pulses. Instruments and Experimental Techniques, 42, 142-144. 